\title{
Evidence for Internal Field in Graphite: a Conduction Electron Spin Resonance Study
}

\author{
M.S. Sercheli, Y. Kopelevich, R. Ricardo da Silva*, J.H.S. Torres, and C. Rettori, \\ Instituto de Física “Gleb Wataghin”, UNICAMP,13083-970, Campinas-SP, \\ Brazil.
}

(July 5, 2021)

\begin{abstract}
We report conduction electron spin resonance measurements performed on highly oriented pyrolitic graphite samples between $10 \mathrm{~K}$ and $300 \mathrm{~K}$ using S $(\nu=4 \mathrm{GHz}), \mathrm{X}(\nu=9.4 \mathrm{GHz})$, and $\mathrm{Q}(\nu=34.4 \mathrm{GHz})$ microwave bands for the external $d c$-magnetic field applied parallel $(H \| c)$ and perpendicular $(H \perp c)$ to the sample hexagonal $c$-axis. The results obtained in the $H \| c$ geometry are interpreted in terms of the presence of an effective internal ferromagnetic-like field, $H_{\text {int }}^{e f f}(T, H)$, that increases as the temperature decreases and the applied $d c$-magnetic field increases. We associate the occurrence of the $H_{\text {int }}^{\text {eff }}(T, H)$ with the field-induced metal-insulator transition in graphite and discuss its origin in the light of relevant theoretical models. 76.30.Pk,71.30.+h,71.27.+a
\end{abstract}


Recent experiments revealed a number of unexpected phenomena in graphite such as a linear energy dependence of the quasiparticle damping, [1] high- $T$ superconducting and ferromagnetic (FM) correlations, [2,3] and magnetic-field-induced metal-insulator transition (MIT). [2,4] These properties could be understood taking into account electron-electron interactions within the graphite layers. [5] A possible proximity of graphite monolayer to the excitonic instability was an alternative explanation for both, the linear energy dependence of the quasiparticle damping and the weak FM [6]. These observations bring graphite to the class of materials which attract a broad scientific interest, including high- $T_{c}$ superconducting (HTS) cuprates, in which a strong Coulomb repulsion between carriers is the key issue, [7] hexaborides demonstrating a high- $T$ weak FM, [8] and most recently discovered superconducting $\mathrm{MgB}_{2}$, [9] a material isoelectronic to graphite. [10]

It has been proved long ago [11] that the electron spin resonance (ESR) is a powerful experimental technique that allows to probe directly the properties of conduction electrons (holes) in graphite. However, the observed $g$-factor anisotropy and its temperature dependence remains unexplained by existing theories which consider the spin-orbit coupling mechanism, [1] indicating the necessity of further experimental and theoretical work.

In this letter we report the experimental results which provide a fresh insight on the conduction ESR (CESR) in highly oriented pyrolitic graphite (HOPG) samples. In particular, we show that the results can be interpreted in terms of an effective internal FM-like field, $H_{\text {int }}^{\text {eff }}(T, H)$, induced by the external $d c$-magnetic field, $H_{\text {ext }}=H$, applied along the sample hexagonal $c$-axis. The results obtained in this work indicate that the occurrence of $H_{\text {int }}^{e f f}(T, H)$ intimately couples to the magnetic-field-induced MIT.

CESR measurements performed on three HOPG samples, synthesized at the Research Institute "Graphite" (Moscow) [2] and the Union Carbide Co. were carried out in a temperature interval from $10 \mathrm{~K}$ to $300 \mathrm{~K}$ by means of a Bruker ELEXSYS-CW spectrometer operating at three microwave frequency bands, $\mathrm{S}(\nu=4 \mathrm{GHz}), \mathrm{X}(\nu=9.4 \mathrm{GHz})$, and $\mathrm{Q}(\nu$ $=34.4 \mathrm{GHz}$ ). The measurements with mutually perpendicular microwave and $d c$-magnetic fields were performed in both $H \| c$ and $H \perp c$ configurations. In order to warrant a 
maximal microwave penetration and to avoid a line-shape change caused by anisotropic skin depth effects, [12] the microwave field was always kept perpendicular to the sample $c$-axis. Similar results were obtained for all graphite samples studied in this work. Complementary low-frequency $(\nu=1 \mathrm{~Hz})$ standard four-probe basal-plane resistance $R(T, H)$ and $d c$-Hall effect measurements were performed for $H \| c$ using a Quantum Design PPMS-9 T and Janis-9 T magnet systems.

The obtained low field in-plane carrier mobility $\sim 7 \cdot 10^{5}-2 \cdot 10^{6} \mathrm{~cm}^{2} / \mathrm{V} \cdot \mathrm{s}(T=4.2$ K) indicates the high quality of our samples. [11] The high sample quality has also been confirmed by means of Shubnikov-de Hass oscillations measurements, where, e.g., two resistance minima at $H=6.7 \mathrm{~T}$ and $H=7.5 \mathrm{~T}$, corresponding to the spin splitting of the $n=1$ Landau level for electrons, 13 15 could clearly be resolved.

In all our CESR measurements a Dysonian line-shape with $A / B$ asymmetry ratio between 4 and 9 was observed. [16] The CESR parameters (resonance field and line-width) were obtained assuming an admixture of absorption/dispersion of a Lorentzian line. [17] The integrated absorption part of the resonance was found to be $T$-independent within the accuracy of the measurements, confirming that the ESR originates from itinerant carriers. 18, 19,

Figure 1 (a, b, c) presents the $T$-dependencies of the resonance field $H_{R}(T)$ for one of our studied samples (HOPG-3, see Ref. [4]) in $H \| c$ and $H \perp c$ geometry using $\mathrm{S}, \mathrm{X}$, and $\mathrm{Q}$ bands, respectively. As can be seen from Fig. $1, H_{R}$ obtained for $H \perp c$ is $T$ independent within the experimental resolution, whereas $H_{R}(T)$ measured for $H \| c$ is a decreasing function of $T$. Note, that $H_{R \|}-H_{R \perp}<0$ which can be re-written in terms of the Lande $g$-factor shift $\Delta g=g_{\|}-g_{\perp}>0$. This is a well known experimental fact being, however, in conflict with the theoretical predictions of $\Delta g<0$ based on the spinorbit interaction. [11] Figure 2 shows $T$-dependencies of the difference $\Delta H_{R}=H_{R \perp}-H_{R \|}$ extracted from the data of Fig. 1. These data demonstrate that $\Delta H_{R}(T, H)$ increases as the temperature decreases and the measuring field (frequency) increases. The inset in Fig. 2 depicts the behavior of $g_{\|}$also extracted from the data of Fig. 1. As can be seen from this 
plot, the $g_{\|}(T, H)$ is both $T$ - and $H$-dependent. Note a rather complex and non-systematic behavior of $g_{\|}(T, H)$ : while $g_{\|}(T, H)$ obtained from the $\mathrm{S}$ band data coincides with that obtained from $\mathrm{Q}$ band for $T \gtrsim 70 \mathrm{~K}$, it coincides with the $g_{\|}(T, H)$ measured with $\mathrm{X}$ band for $T<70 \mathrm{~K}$, and all three bands give the same value of the $g$-factor at $T=300 \mathrm{~K}$ (see inset in Fig. 2). On the other hand, a comparative analysis of the CESR and transport measurements revealed an intriguing correlation between $\Delta H_{R}(T, H)$ and the basal-plane resistance, $R(T, H)$, suggesting that the description of the results presented in Fig. 1 in terms of $\Delta H_{R}(T, H)$ is more appropriate than that in terms of $g_{\|}(T, H)$. It is shown in Fig. 2 that $\Delta H_{R}(T, H)$ can be well approximated by the empirical equation:

$$
\Delta H_{R}(T, H)=H_{0} \ln \left[R(T, H) / R_{0}(H)\right]
$$

where $H_{0}$ and $R_{0}(H)$ are fitting parameters. Both, $\Delta H_{R}(T, H)$ and $R(T, H)$ decrease approaching the transition from insulating-like $(d R / d T<0)$ to the metallic-like $(d R / d T>0)$ resistance behavior, [4 implying that the appearance of the $\Delta H_{R}(T, H)>0$ is essentially coupled to the field-induced MIT.

It is already established that the CESR results of Fig. 1 are characteristic of both HOPG and single crystalline graphite. [11] Because this and in order to be more specific on our claim regarding the relationship between $\Delta H_{R}(T, H)$ and MIT, we present in Fig. $3(\mathrm{a}, \mathrm{b})$ the $R(T, H)$ data obtained for HOPG (Union Carbide Co.) and Kish single crystalline graphite samples. The data of Fig. 3 (a, b) provide an unambiguous evidence that the field-induced MIT is an intrinsic property of graphite. We note that the low temperature leveling-off of the insulating-like $R(T, H)$ is associated with the reentrant transition to the metallic state, [2] which will be not discussed in the present work. A careful analysis of the results given in Fig. 3 (a, b) revealed that the insulating-like resistance $R(T, H)$ develops below a well defined field-dependent temperature $T_{\min }(H)$ which is an increasing function of $H$. This is exemplified for the Kish graphite in Fig. 4 where the reduced resistance $R(T) / R\left(T_{\min }\right)$ vs. $T$ is plotted. The inset in Fig. 4 shows $T_{\min }(H)$ which can be best described (dashed curve) by the formula: 


$$
T_{\min }(H)=A\left(H-H_{c}\right)^{1 / 2}
$$

with $A$ and $H_{c}$ being the fitting parameters. For $H>1 \mathrm{kOe}$, no minimum in $R(T)$ has been found in the studied temperature interval.

In attempts to understand the origin of $\Delta H_{R}(T, H)>0$, which suggests the existence of an effective internal FM-like field $H_{\text {int }}^{\text {eff }}(T, H)$, as well as its coupling to the MIT we note that the characteristic feature of the band structure of a single graphene layer is that there are two isolated points in the first Brillouin zone where the band dispersion is linear $E(\mathbf{k})=$ $\hbar \mathrm{v}|\mathbf{k}|\left(\mathrm{v}=\mathrm{v}_{F} \sim 10^{6} \mathrm{~m} / \mathrm{s}\right.$ is the Fermi velocity $)$, so that the electronic states can be described in terms of the Dirac equations in two dimensions. [5,6.20 Besides, an opening of the gap in the spectrum of Dirac fermions by an applied magnetic field (the so-called "magnetic catalysis" phenomenon), accompanied by the time-reversal symmetry breaking, has been discussed in a number of recent theoretical works (see e.g., [21,22], and references therein). The equation for the field-induced transition temperature $T_{c}(H)$ to the gapped state is given by:

$$
k_{B} T_{c}(H)=C \hbar \mathrm{v}(2 e / \hbar)^{1 / 2}\left(H-H_{c}\right)^{1 / 2},
$$

which is similar to the Eq. (2) derived from the experiment. Here $C$ is the constant, $\Delta_{0}=\hbar \mathrm{v}(2 e / \hbar)^{1 / 2}\left(H-H_{c}\right)^{1 / 2}$ is the induced zero-temperature gap, and $H_{c}$ is the critical field. According to the theory, the development of a magnetic moment proportional to the $\Delta(T, H)$ is also expected. In the context of the present work this would imply $H_{i n t}^{e f f}(T, H) \sim$ $\Delta(T, H)$ which can account for the correlation between $H_{\text {int }}^{e f f}(T, H)$ and $R(T, H)$, see Fig. 2. Whereas in Refs. $[18,19]$ the gap occurrence is related to the development of an additional superconducting order parameter in HTS, the gap in the case of graphite is associated with the insulating-like state. However, as emphasized by Laughlin [21] the theory [Eq. (3)] considers energy scales only. In the light of theoretical results of Ref. [6], it is possible that the here observed phenomenon is related to the field-driven metal-excitonic insulator transition. 
While the analysis of the experimental results in terms of an effective internal FM-like field occurrence appears to be self-consistent, we cannot exclude a complex dependence of the $g$-factor on the applied magnetic field and temperature (see inset in Fig.2) as an alternative approach. However a lack of a proper theory for both magnetic field and temperature dependence [11] of the $g$-factor prevents us to speculate on this matter.

Finally, we present in Fig. 5 the CESR half line-width, $\Delta H_{1 / 2}(T)$, measured at various frequencies $(\nu=4 \mathrm{GHz}, \nu=9.4 \mathrm{GHz}$, and $\nu=34.4 \mathrm{GHz})$. Due to an intrinsic mosaic effect in HOPG the line-width for $H \| c$ is always broader than for $H \perp c$. As can be observed in Fig. 5, $\Delta H_{1 / 2}(T)$ increases with temperature decreasing, in agreement with previous studies. [11] The novel result, however, is that $\Delta H_{1 / 2}(T)$ is essentially field (frequency)-independent. Then, the induced effective internal FM-like field should be highly uniform. In principle, various physical processes such as a distribution of $g$-factor values, motional narrowing, many-body exchange, and relaxation effects can contribute to $\Delta H_{1 / 2}(T)$, [11,23] so that a further work is needed to provide a plausible explanation for $\Delta H_{1 / 2}(T)$. For example, pulsed CESR experiments where the spin-spin and spin-lattice relaxation times can be measured, are highly desirable.

In summary, CESR measurements performed on HOPG samples using $\mathrm{S}(\nu=4 \mathrm{GHz})$, $\mathrm{X}(\nu=9.4 \mathrm{GHz})$, and $\mathrm{Q}(\nu=34.4 \mathrm{GHz})$ microwave bands revealed a complex behavior of the $g$-factor as a function of the applied magnetic field which cannot be understood within a framework of the existing theories. Alternatively, we show that the results can be self-consistently described in terms of the occurrence of an effective internal FM-like field induced by the applied $d c$-magnetic field $(H \| c$-axis). It is also found that the occurrence of the internal field is intimately coupled to the magnetic field-induced MIT. We argue that the magnetic field-induced gap in the energy spectrum of Dirac fermions in graphite can be responsible for the here observed phenomena.

This work is supported by FAPESP, CNPq, and CAPES Brazilian science agencies. We thank V.V. Lemanov and P. Scharff for supplying the graphite samples used in the present 
study, and D.V. Khveshchenko for discussions.

* Also at FAENQUIL, 12600-000, Lorena, SP, Brazil. 


\section{REFERENCES}

[1] S. Xu, J. Cao, C.C. Miller, D.A. Mantell et al., Phys. Rev. Lett. 76, 483 (1996).

[2] Y. Kopelevich, V.V. Lemanov, S. Moehlecke, and J.H.S. Torres, Fizika Tverd. Tela (St. Petersburg) 41, 2135 (1999) [Phys. Solid State 41, 1959 (1999)].

[3] Y. Kopelevich, P. Esquinazi, J.H.S. Torres, and S. Moehlecke, J. Low Temp. Phys. 119, $691(2000)$.

[4] H. Kempa, Y. Kopelevich, F. Mrowka, A. Setzer, et al., Solid State Commun. 115, 539 $(2000)$.

[5] J. Gonzalez, F. Guinea, and M.A.H. Vozmediano, Phys. Rev. Lett. 77, 3589 (1996); Phys. Rev. B 63, 13442 (2001).

[6] D.V. Khveshchenko, cond-mat/0101306.

[7] P.W. Anderson, Science 235, 1196 (1987); Physica C 341, 1575 (2000).

[8] D.P. Young, D. Hall, M.E. Torelli, Z. Fisk et al., Nature 397, 412 (1999).

[9] J. Nagamatsu, N. Nakagawa, T. Muranaka, Y. Zenitani, and J. Akimitsu, Nature 410, $63(2001)$.

[10] G. Baskaran, cond-mat/0103308.

[11] M.S. Dresselhaus, G. Dresselhaus, K. Sugihara, I.L. Spain, and H.A. Goldberg in: "Graphite Fibers and Filaments", Springer Series in Material Science Vol. 5, pp. 179188 (Springer-Verlag, Berlin, N. Y., London, Paris, Tokyo), and references therein; M.S. Dresselhaus and G. Dresselhaus, Adv. Phys. 30, 139 (1981).

[12] L. Walmsley, G. Ceotto, J.H. Castilho, and C. Rettori, Synthetic Metals 30, 97 (1989).

[13] Y. Iye, P.M. Tedrow, G. Timp, M. Shayegan et. al., Phys. Rev. B 25, 5478 (1982).

[14] G. Timp, P.D. Dresselhaus, T.C. Chieu, G. Dresselhaus, and Y. Iye, Phys. Rev. B 28, 
$7393(1983)$.

[15] Y. Iye, L.E. McNeil, and G. Dresselhaus, Phys. Rev. B 30, 7009 (1984).

[16] F.J. Dyson, Phys. Rev. 98, 349 (1955); G. Feher and A.F. Kip, Phys. Rev. 98, 337 (1955).

[17] G.E. Pake and E.M. Purcell, Phys. Rev. 74, 1184 (1948).

[18] A. Abragam and B. Bleaney, EPR of Transition Ions (Clarendon Press, Oxford, 1970).

[19] R.H. Taylor, Adv. in Physics 24, 681 (1975); S. E. Barnes, ibid. 30, 801 (1981).

[20] L. Balents and M.P.A. Fisher, Phys. Rev. B 55, 11973 (1997).

[21] R.B. Laughlin, Phys. Rev. Lett. 80, 5188 (1998).

[22] E.J. Ferrer, V.P. Gusynin, and V. de la Incera, cond-mat/0101308.

[23] D. Lubzens, M.R. Shanabarger, and S. Schultz, Phys. Rev. Lett. 29, 1387 (1972); D. Lubzens and S. Schultz, Phys. Rev. Lett. 36, 1104 (1976). 


\section{FIGURES}

FIG. 1. T-dependencies of the resonance field, $H_{R}(T)$, measured in the HOPG-3 sample for $H \| c$ (squares) and $H \perp c$ (triangles) geometries using $\mathrm{S}(\nu=4 \mathrm{GHz}), \mathrm{X}(\nu=9.4 \mathrm{GHz})$, and $\mathrm{Q}(\nu=34.4 \mathrm{GHz})$ microwave bands. The indicated $g$-factors correspond to the values at room- $T$. The continuous lines are guide for the eye.

FIG. 2. $\Delta H_{R}(T)$ obtained from the data of Fig. 1 (solid symbols). Open symbols correspond to $\Delta H_{R}(T)$ calculated from Eq. (1) using basal-plane resistance data $(H \| c)$ obtained for the HOPG-3 sample with $H=1.34 \mathrm{kOe},(\triangle), H=3.14 \mathrm{kOe}(\square)$, and $H=11.5 \mathrm{kOe}(\circ)$ and fitting parameters $H_{0}=150$ Oe, $R_{0}=0.014 \Omega ; H_{0}=150$ Oe, $R_{0}=0.01 \Omega ; H_{0}=150$ Oe, $R_{0}=0.004$

$\Omega$, respectively. Inset depicts $g_{\|}(T, H)$ extracted from the $H_{R}(T, H)$ data given in Fig. 1 . The continuous lines are guide for the eye.

FIG. 3. Basal-plane resistance, $R(T, H)$, measured at various magnetic fields (in kOe) applied parallel to the sample $c$-axis in (a) HOPG (Union Carbide Co.) and (b) single crystalline Kish graphite.

FIG. 4. Reduced basal-plane resistance $R(T) / R\left(T_{\min }\right)$ measured in Kish graphite single crystal with $H=0.04 \mathrm{~T}(\square), H=0.05 \mathrm{~T}(\circ), H=0.075 \mathrm{~T}(\triangle)$, and $H=0.1 \mathrm{~T}(\nabla)$ applied parallel to the $c$-axis, where $T_{\min }(H)$, denoted by arrows, is the field-dependent temperature separating insulating-like $\left(T<T_{\min }\right)$ and metallic-like $\left(T>T_{\min }\right)$ resistance behavior. The continuous lines are guide for the eye. Inset shows $T_{\min }(H)$; the dashed line is obtained from Eq. (2) with the fitting parameters $A=350 \mathrm{~K} / \mathrm{T}^{1 / 2}$ and $H_{c}=0.038 \mathrm{~T}$.

FIG. 5. The CESR half line-width, $\Delta H_{1 / 2}(T)$, measured in the HOPG-3 sample for both $H \| c$ and $H \perp c$ geometries using $\mathrm{S}(\nu=4 \mathrm{GHz}), \mathrm{X}(\nu=9.4 \mathrm{GHz})$, and $\mathrm{Q}(\nu=34.4 \mathrm{GHz})$ microwave bands. The dotted lines are guide for the eye. 


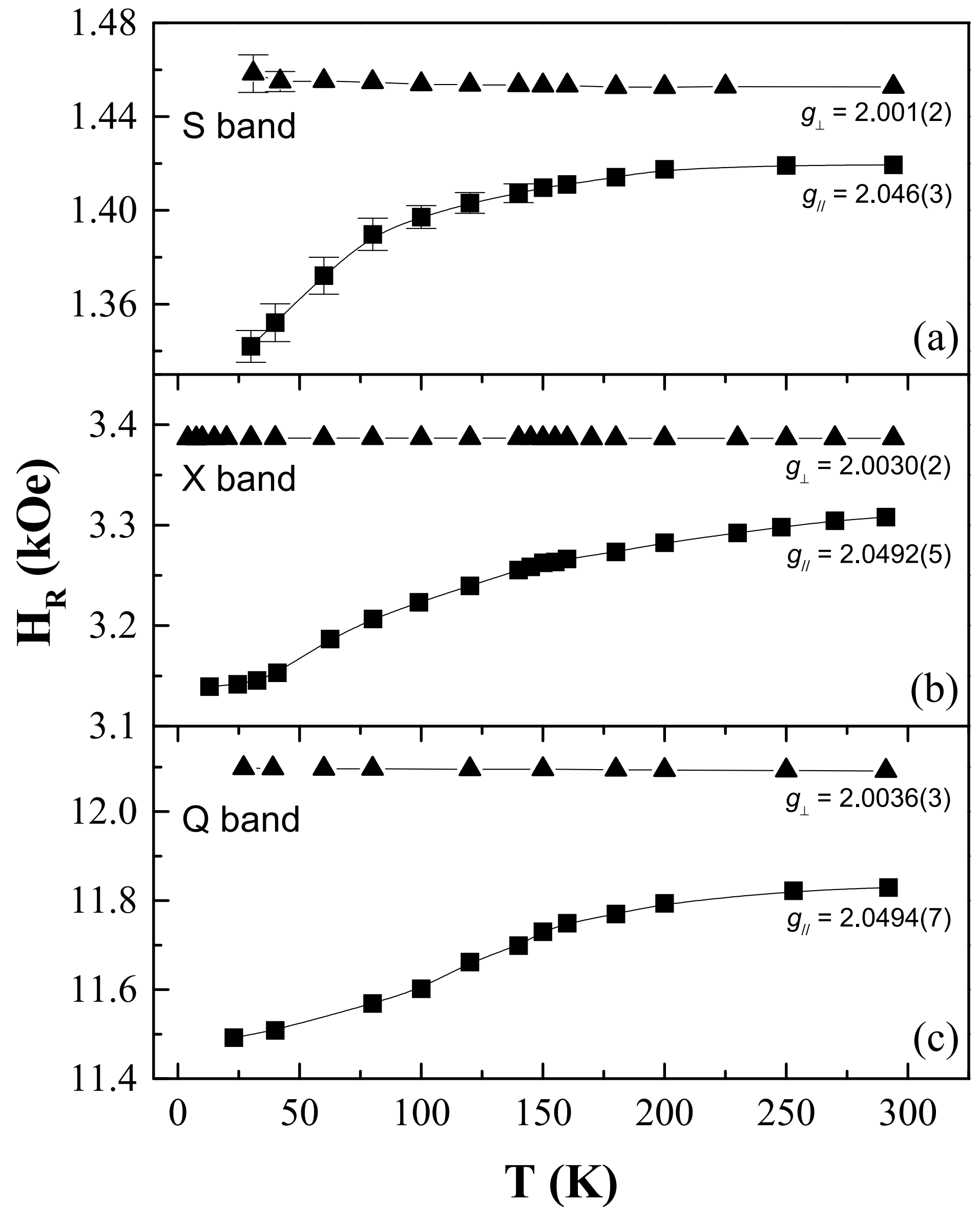




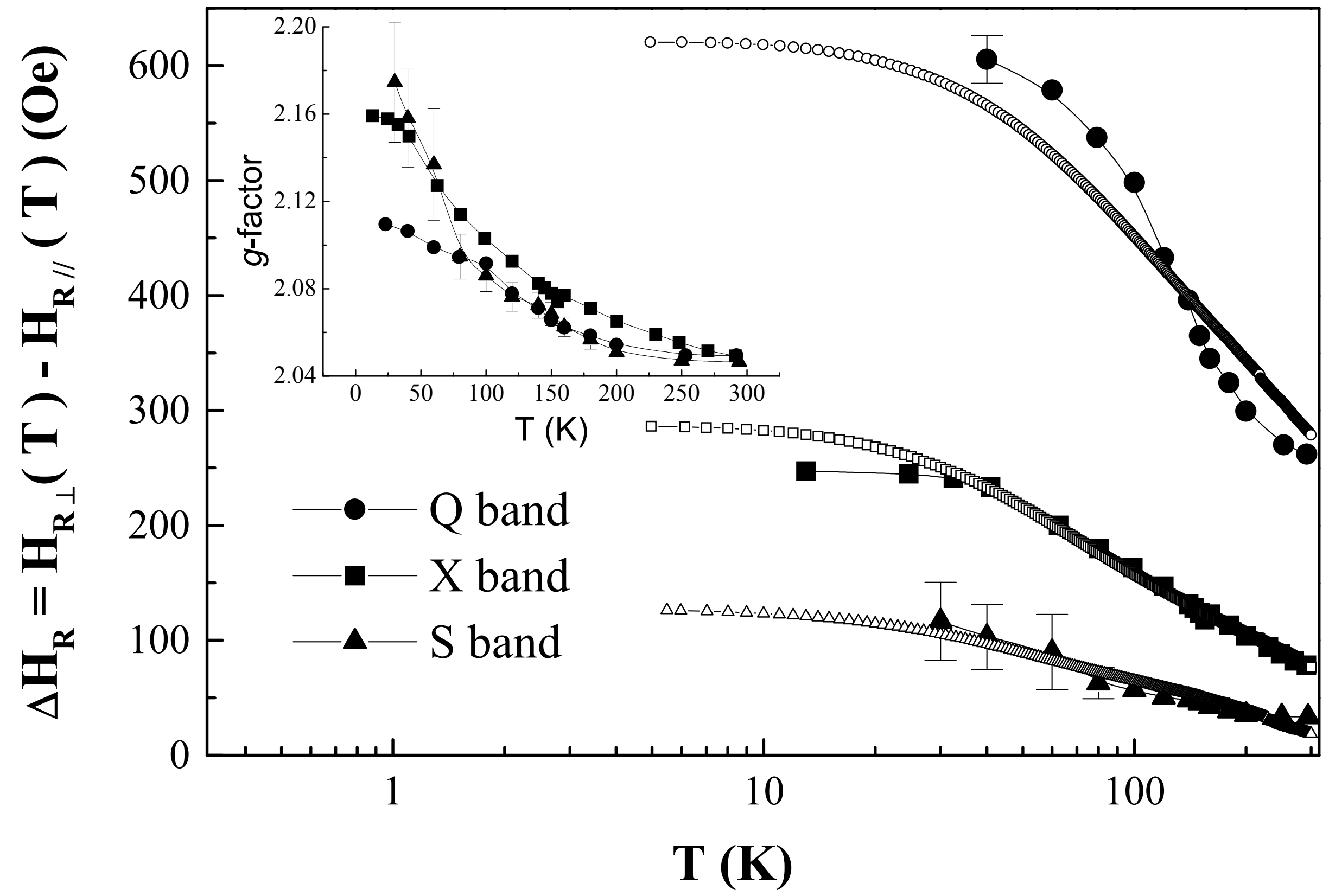




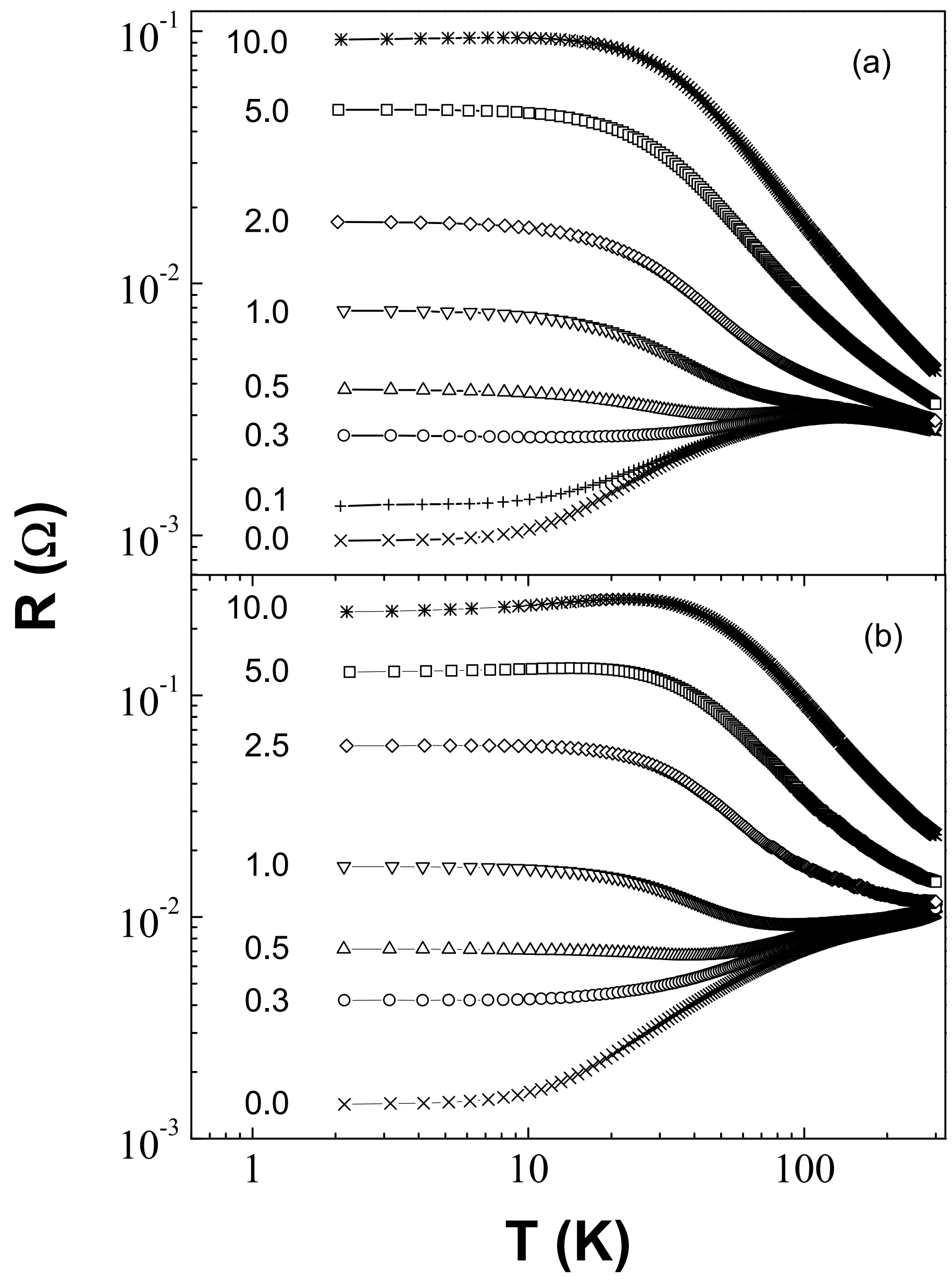




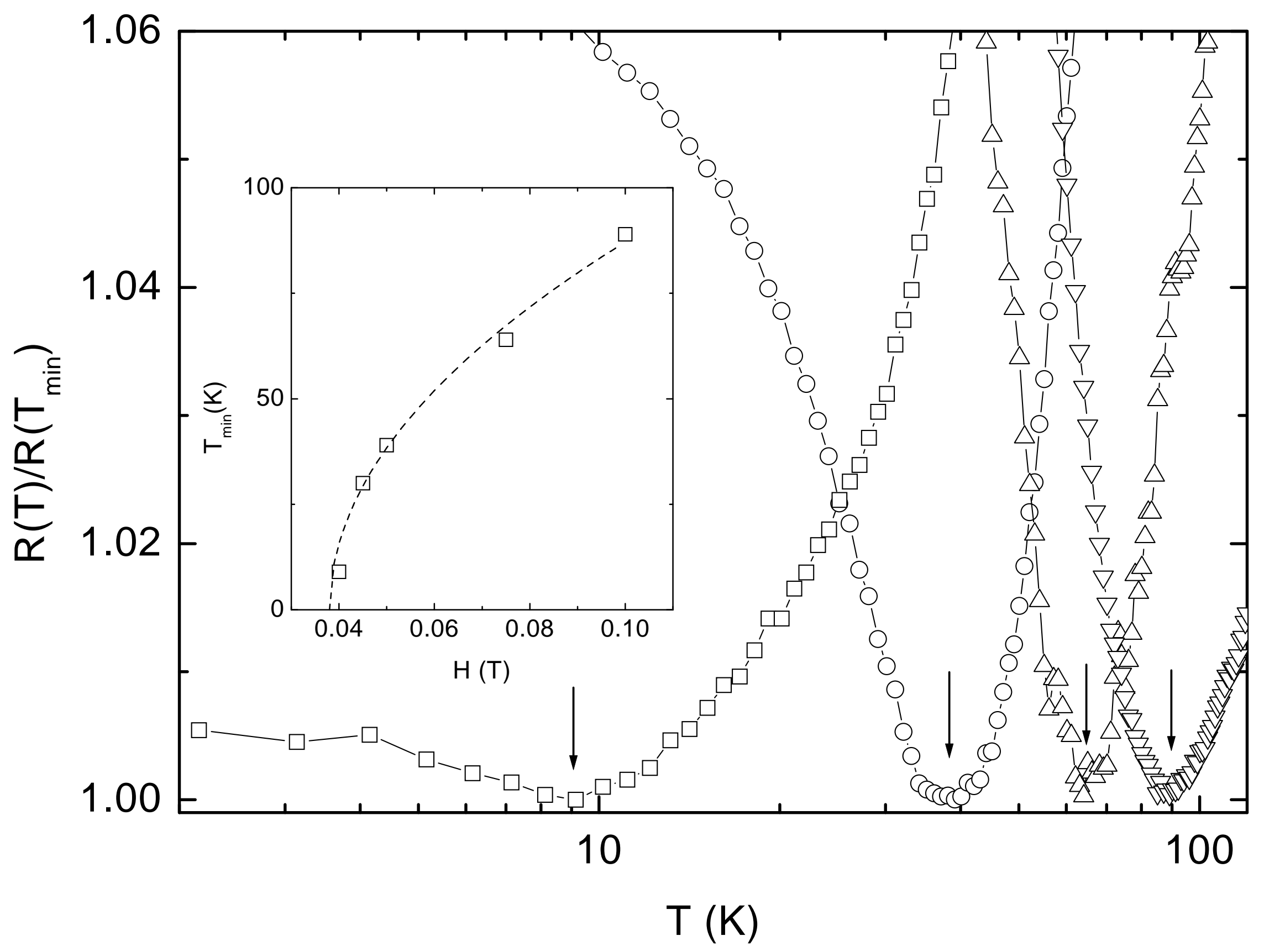




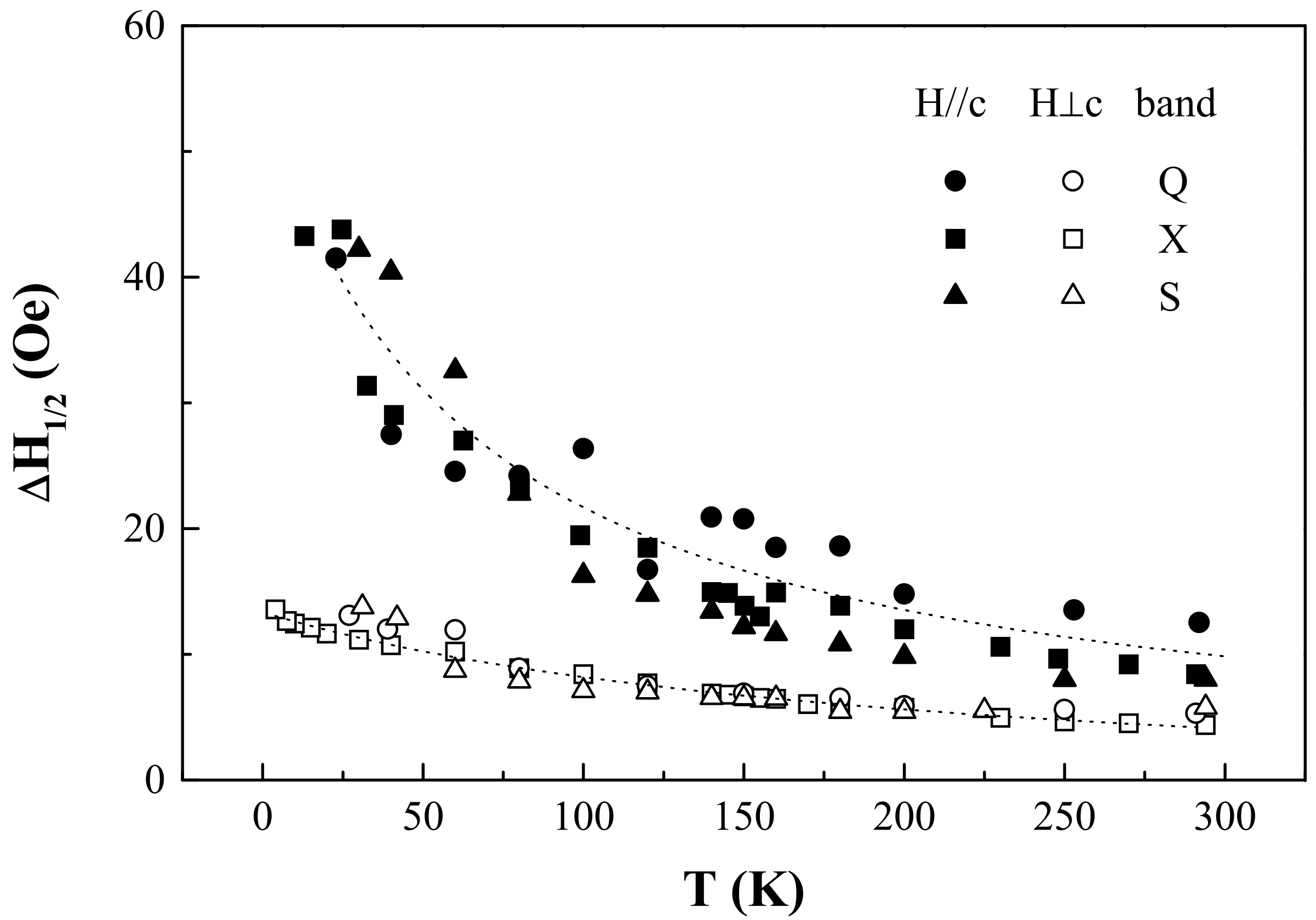

